\title{
Macroscopic approach to N-qudit systems
}

\author{
C. Muñoz, I. Sainz, A.B. Klimov
}

\begin{abstract}
We develop a general scheme for an analysis of macroscopic qudit systems: a) introduce a set of collective observables, which characterizes the macroscopic properties of qudits in an optimal way; b) construct projected $\tilde{Q}$-functions for $N$ qudit systems, containing full macroscopic information; c) propose a collective tomographic protocol both for a general and symmetric $N$-qudit states. The example of $N$-qutrit is analyzed in details and compared to $N$-qubit case.
\end{abstract}




\section{INTRODUCTION}

Basic problems arising in the analysis of macroscopic quantum systems: a) optimization of tomographic schemes in order to reduce the number of measurements [1], [2], [3]; b) processing of available data so that a meaningful information about the state of the system can be efficiently extracted [4]. Both of these tasks are rather complicated since the number of parameters (the number of measurement outcomes) required for a complete characterization of a multipartite quantum state grows exponentially with the number of particles. An intuitively appealing attempt to employ the statistical description of $N$-qudit ( $d$-level) system, by mapping qudits states into distributions in a finite-dimensional grid [5], [6], [7], results inconvenient in the large $N$ limit due to an overwhelming complexity of the resulting discrete functions. Even in the simplest case of $N$-qubit system, the distributions corresponding to relatively simple quantum states have rather complicated structure (usuall represented in form of randomly located peaks [8]]). In addition, the distributions in discrete phase-space do not have natural macroscopic limits, i.e. they do not acquire smooth shapes in the limit $N \gg 1$, which makes very difficult to study their analytical porperties.

While $\sim d^{2 N}$ parameters are needed for a full microscopic description of a generic $N$ qudit state, the global properties can be captured by a significantly smaller number of collective observables. These observables are invariant under particle permutations and thus provide only partial information about the system. On the other hand, frequently only this type of symmetric correlation functions can be efficiently assessed (e.g. due to particle indistinguishability) in macroscopic quantum systems [9].

A quantum state characterization is strongly connected to the specific set of the measurable collective variables. In $N$ qubit systems, the moments of the collective spin operators

$$
S_{x, y, z}=\sum_{i=1}^{N} \sigma_{x, y, z}^{(i)},
$$

provide the global information, allowing to access all $S U(2)$ invariant subspaces appearing in the tensor decomposition $\mathcal{H}_{2}^{\otimes N}$ (without distinguishing subspaces of the same dimension). The results of such collective measurements can used for density matrix estimation [10].

From an analytical perspective, the macroscopic features of an $N$ qubit system are completely described by the so-called projected $\tilde{Q}$-function, defined in a three-dimensional dis-

crete space. This $\tilde{Q}$-function contains full and non-redundant information about results of 
any collective measurement in an arbitrary (not necessarily symmetric) state [11], [12], and allows to outline collective kinematic and dynamic properties in the limit of large number of qubits.

The situation is more complicated for macroscopic systems containing a large number of qudits. Although qudit systems seem to be useful in quantum information protocols [13], only a limited number of experimental reconstructions of one- and bi-partite qudit systems were reported [14] - [22]. Extension to multipartite qudit systems is quite challenging due to a rapid growth of the number of required experimental setups [23], and the deficiency of appropriate theoretical tools to deal with $N \gg 1$ qudit systems. In addition, in this case (in contrast to qubit systems) there are several ways to choose a basis set of symmetric (permutationally invariant) operators, while only some specific sets are convenient for studying macroscopic properties of large number of qudits.

In the present paper we develop a general framework for the analysis of global characteristics of multipartite qudit systems. We will: a) construct projected $\tilde{Q}$-functions for $N$ qudit systems, containing full macroscopic information; b) introduce a set of collective observables, which characterizes macroscopic properties of qudits and compatible with the phase-space description; c) propose two discrete collective tomographic protocols (for general and symmetric $N$-qudit states) and provide an explicit (partial) reconstruction expression for the $N$-qudit density matrix in terms of average values of correlation functions of $\sim N^{d^{2}-1}$ appropriate collective qudit operators. The example of $N$ qutrits will be analyzed in details and compared to $N$-qubit systems.

\section{N QUDIT PROJECTED $\widetilde{Q}$-FUNCTION}

Let us consider $N$-qudit Hilbert space $\mathcal{H}_{d^{N}}=\mathcal{H}_{d}^{\otimes N}$, where $d$ is a prime number, spanned by the computational basis $|\lambda\rangle=\left|l_{1}, \ldots, l_{N}\right\rangle, l_{i} \in \mathbb{Z}_{d}$. The standard unitary $N$-qudit operators are defined according to [6], [24]

$$
Z_{\alpha}=\sum_{\lambda} \omega^{\lambda \alpha}|\lambda\rangle\left\langle\lambda\left|, \quad X_{\beta}=\sum_{\lambda}\right| \lambda+\beta\right\rangle\langle\lambda|
$$

where

$$
\alpha=\left(a_{1}, \ldots, a_{N}\right), \quad \beta=\left(b_{1}, \ldots, b_{N}\right), \quad a_{i}, b_{i}=0, \ldots, d-1,
$$


are $d$ - strings with elements from $\mathbb{Z}_{d}, \alpha \beta=a_{1} b_{1}+\ldots+a_{N} b_{N}, \omega=\exp (2 \pi i / d)$ sums and multiplications are taken by mod $d$. The operators (2) are factorized into tensor products,

of single qudit Pauli operators [25]

$$
Z_{\alpha}=\bigotimes_{i=1}^{N} Z_{i}^{a_{i}}, \quad X_{\beta}=\bigotimes_{i=1}^{N} X_{i}^{b_{i}}
$$

$$
Z_{i}=\sum_{l=0}^{d-1} \omega^{l}|l\rangle\left\langle l\left|, \quad X_{i}=\sum_{l=0}^{d-1}\right| l+1\right\rangle\langle l|,
$$

and satisfy the commutation relation

$$
Z_{\alpha} X_{\beta}=\omega^{\alpha \beta} X_{\beta} Z_{\alpha}
$$

Operators acting in $N$-qudit Hilbert space can be mapped into functions labeled by a pair of $N$-tuples $(\alpha, \beta)$. Two mutually dual maps, known as discrete $Q$-symbols and $P$-symbols [7], [8], [26], have the form

$$
\begin{aligned}
Q_{f}(\alpha, \beta) & =\operatorname{Tr}\left(\hat{\Delta}^{(-1)}(\alpha, \beta) \hat{f}\right), \quad P_{f}(\alpha, \beta)=\operatorname{Tr}\left(\hat{\Delta}^{(1)}(\alpha, \beta) \hat{f}\right), \\
\operatorname{Tr}\left(\hat{\Delta}^{(1)}(\alpha, \beta) \hat{\Delta}^{(-1)}\left(\alpha^{\prime}, \beta^{\prime}\right)\right) & =\delta_{\alpha \alpha^{\prime}} \delta_{\beta \beta^{\prime}}
\end{aligned}
$$

see (103) for the explicit form of the kernels $\hat{\Delta}^{( \pm 1)}$. The first rank projectors

$$
\begin{aligned}
\hat{\Delta}^{(-1)}(\alpha, \beta) & =|\alpha, \beta\rangle\langle\alpha, \beta|, \\
|\alpha, \beta\rangle & =Z_{\alpha} X_{\beta}|\xi\rangle=\bigotimes_{i=1}^{N}\left|a_{i}, b_{i}\right\rangle,
\end{aligned}
$$

form an informationally complete set,

$$
\sum_{\alpha, \beta} \hat{\Delta}^{(-1)}(\alpha, \beta)=d^{N} \hat{I}
$$

The fiducial state $|\xi\rangle$ in (10) is chosen in a product form,

$$
|\xi\rangle=\bigotimes_{i=1}^{N}|\xi\rangle_{i}
$$

in such a way that the single particle projectors on the states

$$
\left|a_{i}, b_{i}\right\rangle=Z_{i}^{a_{i}} X_{i}^{b_{i}}|\xi\rangle_{i}
$$


satisfy the symmetric informationally complete (SIC) POVM condition [27]

$$
\left|\left\langle a_{i}, b_{i} \mid a_{i}^{\prime}, b_{i}^{\prime}\right\rangle\right|^{2}=\frac{1+d \delta_{a_{i}, a_{i}^{\prime}} \delta_{b_{i}, b_{i}^{\prime}}}{1+d}
$$

An operator $\hat{f}$ can be decomposed on the basis of $\hat{\Delta}^{( \pm 1)}(\alpha, \beta)$ as

$$
\begin{aligned}
\hat{f} & =\sum_{\alpha, \beta} Q_{f}(\alpha, \beta) \hat{\Delta}^{(1)}(\alpha, \beta) \\
& =\sum_{\alpha, \beta} P_{f}(\alpha, \beta) \hat{\Delta}^{(-1)}(\alpha, \beta),
\end{aligned}
$$

so that the average values are computed according to

$$
\langle\hat{f}\rangle=\sum_{\alpha, \beta} Q_{\rho}(\alpha, \beta) P_{f}(\alpha, \beta)=\sum_{\alpha, \beta} Q_{f}(\alpha, \beta) P_{\rho}(\alpha, \beta) .
$$

It follows from (9) and (12) that

$$
\mathcal{P} \hat{\Delta}^{( \pm 1)}(\alpha, \beta) \mathcal{P}^{\dagger}=\hat{\Delta}^{( \pm 1)}(\pi \alpha, \pi \beta)
$$

where $\mathcal{P}$ is the permutation operator and $\pi \alpha$ is a permutation of the string $\alpha$ corresponding to $\mathcal{P}$.

Then, $P$ and $Q$ - symbols of permutationally invariant operators, $\hat{s}=\mathcal{P} \hat{s} \mathcal{P}^{\dagger}$, are symmetric functions of their arguments, and thus depend only on the corresponding weights $\mathbf{h}(\alpha, \beta)$ [28] (see also Appendix A)

$$
P_{s}(\alpha, \beta)=\operatorname{Tr}\left(\hat{\Delta}^{(1)}(\alpha, \beta) \hat{s}\right) \equiv P_{s}(\mathbf{h}(\alpha, \beta)) .
$$

The weights $h(\alpha)$, being invariant under permutations characteristics of $d$-strings (3), $h(\alpha)=h(\pi \alpha)$, are defined according to

$$
h(\alpha)=\sum_{i=1}^{N} a_{i}=\sum_{k \in \mathbb{Z}_{d}} k \sum_{i=1}^{N} \delta_{a_{i}, k}, 0 \leq h(\alpha) \leq(d-1) N,
$$

and can be arranged in a vector

$$
\mathbf{h}(\alpha, \beta)=\left\{h(k \alpha+l \beta) ; k, l \in \mathbb{Z}_{d}\right\}
$$

The $d^{2}-1$ components of the vector $\mathbf{h}(\alpha, \beta)$ form a basis in the space of symmetric functions constructed on $(\alpha, \beta)$.

For instance, in case of qubits the three-dimesional $\mathbf{h}$ - vector has the form 


$$
\mathbf{h}(\alpha, \beta)=\{h(\alpha), h(\beta), h(\alpha+\beta)\},
$$

while for qutrits $\mathbf{h}$ - vector is eight-dimensional,

$$
\mathbf{h}(\alpha, \beta)=\{h(\alpha), h(2 \alpha), h(\beta), h(2 \beta), h(\alpha+\beta), h(2 \alpha+2 \beta), h(2 \alpha+\beta), h(\alpha+2 \beta)\}
$$

The points of two-dimensional grid $(\alpha, \beta)$ can be partially ordered according to the values of the weights (21) and an arbitrary function constructed on $(\alpha, \beta)$ satisfy the following summation rule

$$
\sum_{\alpha, \beta} f(\alpha, \beta)=\sum_{\mathbf{m}} \sum_{\alpha, \beta} \delta_{\mathbf{h}(\alpha, \beta), \mathbf{m}} f(\alpha, \beta),
$$

where the components of the vector $\mathbf{m}$ take values from 0 to $(d-1) N$.

Taking into account (19) and the summation rule (24) we observe that the average value of any permutationally invariant operator in an arbitrary (not necessarily symmetric) state is computed according to

$$
\langle\hat{s}\rangle=\sum_{\alpha, \beta} P_{s}(\alpha, \beta) Q_{\rho}(\alpha, \beta)=\sum_{\mathbf{m}} P_{s}(\mathbf{m}) \widetilde{Q}_{\rho}(\mathbf{m}),
$$

where

$$
\begin{aligned}
\widetilde{Q}_{\rho}(\mathbf{m}) & =\sum_{\alpha, \beta} Q_{\rho}(\alpha, \beta) \delta_{\mathbf{h}(\alpha, \beta), \mathbf{m}}=\operatorname{Tr}\left(\hat{\Delta}^{(-1)}(\mathbf{m}) \hat{\rho}\right) . \\
\hat{\Delta}^{( \pm 1)}(\mathbf{m}) & =\sum_{\alpha, \beta} \hat{\Delta}^{( \pm 1)}(\alpha, \beta) \delta_{\mathbf{h}(\alpha, \beta), \mathbf{m}}
\end{aligned}
$$

For symmetric states, $\mathcal{P} \hat{\rho}_{s} \mathcal{P}^{\dagger}=\hat{\rho}_{s}$, where $Q_{\rho_{s}}(\alpha, \beta) \equiv Q_{\rho_{s}}(\mathbf{h}(\alpha, \beta))$, one has

$$
\begin{aligned}
\widetilde{Q}_{\rho_{s}}(\mathbf{m}) & =Q_{\rho_{s}}(\mathbf{h}(\alpha, \beta)) R_{\mathbf{m}}^{(d)} \\
R_{\mathbf{m}}^{(d)} & =\sum_{\alpha, \beta} \delta_{\mathbf{h}(\alpha, \beta), \mathbf{m}}=\sum_{\alpha, \beta} \prod_{\substack{k, l=0 \\
k, l\} \neq\{0,0\} \\
d-1 \\
h(k \alpha}}^{d-1} \delta_{h(k \alpha+l \beta), m_{k l}}
\end{aligned}
$$

where $R_{\mathbf{m}}^{(d)}$ is the multiplicity of each particular set

$$
\mathbf{m}=\left\{m_{k l}=h(k \alpha+l \beta), 0 \leq m_{k l} \leq(d-1) N, k, l=0, \ldots, d-1\right\}
$$

i.e. is the number of pairs $(\alpha, \beta)$ of $d$-strings characterized by the same vector $\mathbf{m}=\left\{m_{k l}=\right.$ $h(k \alpha+l \beta), k, l=0, \ldots, d-1\}$. 
It follows from (25) that the $\widetilde{Q}_{\rho}(\mathbf{m})$-function contains complete and non-redundant information about all macroscopic properties of the state $\hat{\rho}$ and can be considered as a discrete distribution in $d^{2}-1$ dimensional macroscopic measurement space $\mathcal{M}$ spanned by the vectors (30). The total number of multiplets $\{\mathbf{m}=\mathbf{h}(\alpha, \beta)\}$ (points in $\mathcal{M}$ ) is

$$
\mathcal{N}_{\mathcal{M}}=\frac{\left(N+d^{2}-1\right) !}{\left(d^{2}-1\right) ! N !}
$$

which is the amount of collective measurements fully determining the $\widetilde{Q}_{\rho}(\mathbf{m})$ - function. In other words, the global variables "see" an $N$-qudit state in form of $\widetilde{Q}_{\rho}(\mathbf{m})$ distribution in $\mathcal{M}$. In the $N$-qubit case, the $\widetilde{Q}$-function has been extensively studied in [11], [12] and applied to the analysis of pure state thermalization effect and quantum phase transitions [29].

In the macroscopic limit $N \gg d$,

$$
\mathcal{N}_{\mathcal{M}} \sim N^{d^{2}-1} /\left(d^{2}-1\right) !
$$

which is significantly smaller than the number of points in the full discrete phase-space $\sim d^{2 N}$. In practice, distributions corresponding to physically relevant macroscopic states tend to smooth shapes located in certain areas of the measurement space $\mathcal{M}$, as it was observed in qubit case [11], [12], and shown in the following examples.

\section{Examples.}

1. For the fiducial state (12) - (14) one can easily obtain

$$
\widetilde{Q}_{\xi}^{(d)}(\mathbf{m})=(d+1)^{-\frac{2}{d^{2}(d-1)} \sum_{k, l} m_{k l}} R_{\mathbf{m}}^{(d)},
$$

which is a localized distribution (of size $\sim \sqrt{N}$ ) in the $\mathcal{M}$ - space, which tends to the Gaussian shape for $N \gg d$, for instance (see Appendix C). For instance, in N-qubit case $\widetilde{Q}_{\xi}(\mathbf{m})$ is a single "spherically symmetric" Gaussian function centered at $\mathbf{m}_{0}=(3 N / 8,3 N / 8,3 N / 8)$,

$$
\widetilde{Q}_{\xi}^{(2)}(\mathbf{m}) \sim \exp \left(-2 \frac{\left[\left(m_{01}-\frac{3 N}{8}\right)^{2}+\left(m_{01}-\frac{3 N}{8}\right)^{2}+\left(m_{11}-\frac{3 N}{8}\right)^{2}\right]}{N},\right.
$$

while in N-qutri case $\widetilde{Q}_{\xi}(\mathbf{m})$ takes a form of a "squeezed" Gaussian, which can be compactly represented as follows

$$
\widetilde{Q}_{\xi}^{(3)}(\mathbf{m}) \sim \exp \left(-\sum_{i=0}^{1} \sum_{j=0}^{i+1} \frac{\left[\left(m_{i j}-\frac{8 N}{9}\right)^{2}+\left(m_{2 i 2 j}-\frac{8 N}{9}\right)^{2}-\left(m_{i j}-\frac{8 N}{9}\right)\left(m_{2 i 2 j}-\frac{8 N}{9}\right)\right]}{N}\right) .
$$


2. For the $G H Z$-like $N$-qudit state

$$
|G H Z\rangle=\frac{1}{\sqrt{d}} \sum_{l=0}^{d-1}|l \ldots l\rangle .
$$

one obtains by using (10) for the discrete $Q$-function (7) the following explicitly invariant under particle permutations expression

$$
Q(\alpha, \beta)=|\langle G H Z \mid \alpha, \beta\rangle|^{2}=\frac{1}{d}\left|\sum_{l=0}^{d-1} \omega^{l \sum_{i} a_{i}} \prod_{i=1}^{N} c_{l-b_{i}}^{(i)}\right|^{2},
$$

where $a_{i}, b_{i}$ are components of the $d$-string (3) and $c_{l}^{(i)}$ are the expansion coefficients of the $i$-th particle fiducial state in the computational basis,

$$
|\xi\rangle_{i}=\sum_{l=0}^{d-1} c_{l}^{(i)}|l\rangle_{i}
$$

so that

$$
|\xi\rangle=\sum_{\lambda} c_{\lambda}|\lambda\rangle,|\lambda\rangle=\left|l_{1}, . ., l_{N}\right\rangle, c_{\lambda}=\prod_{i=1}^{N} c_{l_{i}}^{(i)},
$$

Thus, according to (28), we arrive at

$$
\widetilde{Q}_{G H Z}^{(d)}(\mathbf{m})=\frac{1}{d}\left|\sum_{l=0}^{d-1} \omega^{m_{l 0}} \prod_{i=1}^{N} c_{l-b_{i}}^{(i)}\right|^{2} R_{\mathbf{m}}^{(d)},
$$

where the product $\prod_{i=1}^{N} c_{l-b_{i}}^{(i)}$ is a function of $m_{0 l}, l=1,2, . ., d-1$ only.

For qubits, $d=2$, and the SIC POVM generating fiducial state

$$
|\xi\rangle_{i}=\frac{|0\rangle_{i}+\zeta|1\rangle_{i}}{\sqrt{1+|\zeta|^{2}}}, \quad \zeta=\frac{\sqrt{3}-1}{\sqrt{2}} e^{i \pi / 4}
$$

the expression (41) is reduced to

$$
\widetilde{Q}_{G H Z}^{(2)}(\mathbf{m})=\frac{1}{2\left(1+|\zeta|^{2}\right)^{N}}\left|\zeta^{m_{01}}+(-1)^{m_{10}} \zeta^{N-m_{01}}\right|^{2} R_{\mathbf{m}}^{(2)},
$$

where $R_{\mathbf{m}}^{(2)}$ is given in (122). The distribution $\widetilde{Q}_{G H Z}^{(2)}(\mathbf{m})$ has a form of two separated by a distance $\sim N$ along the axis $m_{01}$ discrete clouds, each of size $\sim \sqrt{N}$ [11], [12] in three-dimensional measurement space. Each cloud acquires a Gaussian shape centered at $m_{01}=N(1 \pm 1 / \sqrt{3}) / 2$ in the limit $N \gg 1$. In Fig.1 we plot the projection of $\widetilde{Q}_{G H Z}^{(2)}(\mathbf{m})$ on the axis $m_{01}$. there are two maximums at $m_{01}=N(1 \pm 1 / \sqrt{3}) / 2$ 


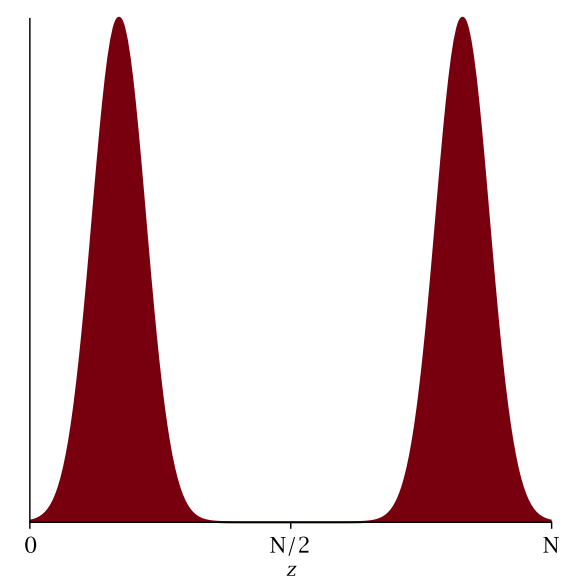

FIG. 1: Projection of $\widetilde{Q}_{G H Z}^{(2)}(\mathbf{m})$ on the axis $m_{01}$.

For qutrits, $d=3$, one obtains

$$
\begin{aligned}
\widetilde{Q}_{G H Z}^{(3)}(\mathbf{m})= & \frac{1}{2^{N} 3} \mid 2^{m_{02} / 3-m_{01} / 6} \delta_{m_{0,1}, 2 m_{02}}+2^{m_{01} / 3-m_{02} / 6}(-\omega)^{m_{01}-N} \delta_{2 m_{01}, m_{02}} \\
& +\left.2^{N / 2-m_{01} / 6-m_{02} / 6}(-\omega)^{m_{01}-m_{20}} \delta_{3 N, m_{01}+m_{02}}\right|^{2} R_{\mathbf{m}}^{(3)}
\end{aligned}
$$

where the fiducial state is chosen as

$$
|\xi\rangle_{i}=\frac{1}{\sqrt{2}}\left(|0\rangle_{i}+e^{i \pi / 3}|1\rangle_{i}\right)
$$

and $R_{\mathbf{m}}^{(3)}$ is given in (124). As it can be appreciated from the above expression, $\widetilde{Q}_{G H Z}^{(3)}(\mathbf{m})$ is a superposition of three localized clusters, each tending to a Gaussian form centered at in the plane $\left(m_{01}, m_{02}\right)$ of eight-dimensional $\mathcal{M}$ - space at $\left(m_{01}, m_{02}\right)=$ $(N, N / 2),(N / 2, N),(3 N / 2,3 N / 2)$.

In qubit case $\widetilde{Q}_{\rho}(\mathbf{m})$ can be plotted as density distributions in a three-dimensional space. For higher dimensions $\widetilde{Q}_{\rho}(\mathbf{m})$ can be represented only in form of projections into hyperplanes in the full $\mathcal{M}$ - space. Nevertheless, the analytical properties of the $\widetilde{Q}$-functions are very useful for analysis of the global properties of macroscopic systems, as it will be shown below. 


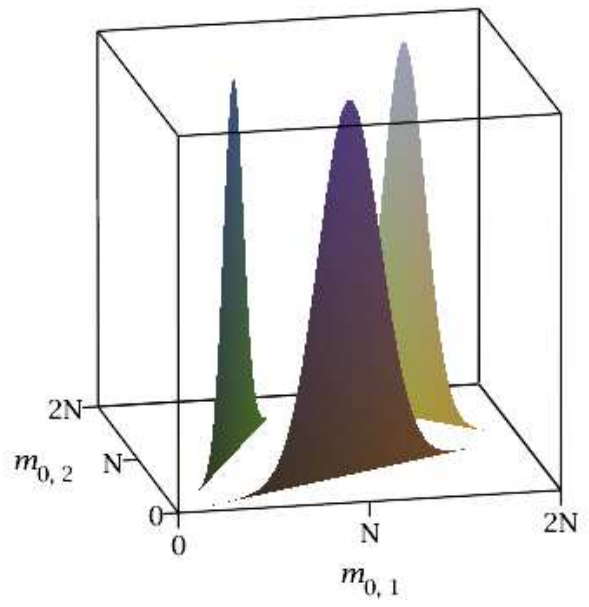

FIG. 2: Projection of $\widetilde{Q}_{G H Z}^{(3)}(\mathbf{m})$ on the plane $m_{01}-m_{02}$.

\section{A. Collective operators}

Let us introduce the following set of collective (invariant under particle permutations) Hermitian operators for an $N$-qudit system

$$
\hat{O}_{k, l}=N \hat{I}-\frac{2}{(d-1) d^{N}} \sum_{\alpha, \beta} h(k \alpha+l \beta)|\alpha, \beta\rangle\langle\alpha, \beta|, \quad k, l=0, \ldots, d-1 .
$$

Their $P$-symbols (7) are proportional to the corresponding weights:

$$
P_{\hat{O}_{k l}}=\frac{1}{d^{N}}\left[N-\frac{2}{d-1} h(k \alpha+l \beta)\right],
$$

which allows to associate the axes in the measurement space $\mathcal{M}$ with the collective operators (46). This can be very helpful for determination of measurements required for a detection

of quantum states. For instance, the main features of the qutrit GHZ-like state (38) can be recognized by measuring $\hat{O}_{0,1}$ and $\hat{O}_{0,2}$ operators and their moments, see Fig.2.

It is proven in Appendix B that the operators (46) are split into $d+1$ disjoint sets of $d$ commuting operators:

$$
\left\{\hat{O}_{\lambda k, \lambda l}, \lambda=1, \ldots, d-1\right\}, \quad\left\{\hat{O}_{0, l}\right\}, \quad\left\{\hat{O}_{k, 0}\right\}, k, l=1, \ldots, d-1
$$

where

$$
\left[\hat{O}_{\lambda k, \lambda l}, \hat{O}_{k, l}\right]=0, \quad\left[\hat{O}_{0, l}, \hat{O}_{0, l^{\prime}}\right]=0, \quad\left[\hat{O}_{k, 0}, \hat{O}_{k^{\prime}, 0}\right]=0
$$


and

$$
\operatorname{tr}\left(\hat{O}_{k, l} \hat{O}_{k^{\prime}, l^{\prime}}\right)=0, \quad k^{\prime} l \neq k l^{\prime} .
$$

The collective observables (46) can be represented in form of a direct product of singleparticle operators

$$
\begin{aligned}
\hat{O}_{k, l} & =\sum_{i=1}^{N} \hat{I} \otimes \ldots \otimes \hat{O}_{k, l}^{(i)} \otimes \ldots \otimes \hat{I}, \\
\hat{O}_{k, l}^{(i)} & =\hat{I}^{(i)}-\frac{2}{d(d-1)} \sum_{a_{i}, b_{i}=0}^{d-1}\left\{k a_{i}+l b_{i}\right\}\left|a_{i}, b_{i}\right\rangle\left\langle a_{i}, b_{i}\right|,
\end{aligned}
$$

where $\left|a_{i}, b_{i}\right\rangle$ are the states (13) and the operations in $\{\cdot\}$ are taken mod $d$. The operators (52) are normalized according to

$$
\operatorname{Tr} \hat{O}_{k, l}^{(i)}=0, \quad \operatorname{Tr}\left(\left[\hat{O}_{k, l}^{(i)}\right]^{2}\right)=\frac{d}{3(d-1)},
$$

and form a basis of the $s u(d)$ algebra similar to that introduced in [30]. The explicit form of the matrix elements of the operators $\hat{O}_{k, l}^{(i)}$ in the computational basis is given in Appendix B, Eqs. (111)-(112).

Taking into account (13) one obtains that $d-1$ operators $\hat{O}_{0, l}^{(i)}$ are diagonal in the logical basis (see Appendix B). The elements of the commuting sets containing non-diagonal operators $\hat{O}_{k, l}^{(i)}, k \neq 0$, are convenient to label as $\hat{O}_{\lambda, \lambda m}^{(i)}$, which matrix elements in the computational basis are given in Appendix B. The commuting sets $\left\{\hat{O}_{\lambda, \lambda m},\left[\hat{O}_{\lambda, \lambda m}, \hat{O}_{\lambda, \lambda m^{\prime}}\right]=0\right.$, $\left.m, m^{\prime}=0, \ldots, d-1\right\}$ are obtained from the diagonal set $\left\{\hat{O}_{0, l}\right\}$ by $S U(d)$ transformations, and thus can be efficiently measured.

\section{Examples}

1. Qubits, $d=2$. The fiducial state (42) leads to the natural representation

$$
\hat{O}_{0,1}^{(i)}=\frac{1}{\sqrt{3}} \sigma_{z}^{(i)}, \quad \hat{O}_{1,0}^{(i)}=\frac{1}{\sqrt{3}} \sigma_{x}^{(i)}, \quad \hat{O}_{1,1}^{(i)}=\frac{1}{\sqrt{3}} \sigma_{y}^{(i)},
$$

so that the operators (46) coincide up to a constant factor with the spin collective operators (1).

2. Qutrits, $d=3$. Taking the fiducial state (45) we obtain the following four commutative 
sets of cyclic (up to a constant) operators, $\hat{O}_{k, l}^{(i)}=4\left(\hat{O}_{k, l}^{(i)}\right)^{3},($ compare to [30] $)$

$$
\begin{aligned}
& \hat{O}_{0,1}^{(i)}=\frac{1}{2}\left[\begin{array}{ccc}
0 & 0 & 0 \\
0 & 1 & 0 \\
0 & 0 & -1
\end{array}\right], \hat{O}_{0,2}^{(i)}=\frac{1}{2}\left[\begin{array}{ccc}
1 & 0 & 0 \\
0 & 0 & 0 \\
0 & 0 & -1
\end{array}\right] \text {, } \\
& \hat{O}_{1,0}^{(i)}=\frac{i}{2 \sqrt{3}}\left[\begin{array}{ccc}
0 & -1 & 1 \\
1 & 0 & -1 \\
-1 & 1 & 0
\end{array}\right], \hat{O}_{2,0}^{(i)}=\frac{1}{2 \sqrt{3}}\left[\begin{array}{ccc}
0 & e^{-i \pi / 6} & e^{i \pi / 6} \\
e^{i \pi / 6} & 0 & e^{-i \pi / 6} \\
e^{-i \pi / 6} & e^{i \pi / 6} & 0
\end{array}\right] \text {, } \\
& \hat{O}_{1,1}^{(i)}=\frac{1}{2 \sqrt{3}}\left[\begin{array}{ccc}
0 & -i & e^{-i 5 \pi / 6} \\
i & 0 & e^{i \pi / 6} \\
e^{i 5 \pi / 6} & e^{-i \pi / 6} & 0
\end{array}\right], \hat{O}_{2,2}^{(i)}=\frac{1}{2 \sqrt{3}}\left[\begin{array}{ccc}
0 & e^{-i \pi / 6} & e^{i 5 \pi / 6} \\
e^{i \pi / 6} & 0 & i \\
e^{-i 5 \pi / 6} & -i & 0
\end{array}\right] \text {, } \\
& \hat{O}_{2,1}^{(i)}=\frac{1}{2 \sqrt{3}}\left[\begin{array}{ccc}
0 & e^{-i \pi / 6} & -i \\
e^{i \pi / 6} & 0 & e^{-i 5 \pi / 6} \\
i & e^{i 5 \pi / 6} & 0
\end{array}\right], \hat{O}_{1,2}^{(i)}=\frac{1}{2 \sqrt{3}}\left[\begin{array}{ccc}
0 & -i & e^{-i \pi / 6} \\
i & 0 & e^{i 5 \pi / 6} \\
e^{i \pi / 6} & e^{-i 5 \pi / 6} & 0
\end{array}\right]
\end{aligned}
$$

The sets (56) - (58) can be obtained form the set (55) by $S U(3)$ rotations.

\section{COLLECTIVE TOMOGRAPHY}

By a direct substitution one can prove that the symmetrized operators (27) form a biorthogonal set

$$
\operatorname{Tr}\left[\hat{\Delta}^{(1)}(\mathbf{m}) \hat{\Delta}^{(-1)}\left(\mathbf{m}^{\prime}\right)\right]=R_{\mathbf{m}}^{(d)} \delta_{\mathbf{m}, \mathbf{m}^{\prime}}
$$

This suggests to approximate the density matrix by "inverting" Eq. (26) in the form similar to Eq. (15)

$$
\hat{\rho} \approx \hat{\rho}_{r e c}=\sum_{\mathbf{m}} \widetilde{Q}_{\rho}(\mathbf{m})\left(R_{\mathbf{m}}^{(d)}\right)^{-1} \hat{\Delta}^{(1)}(\mathbf{m}) .
$$

The above equation is a formal expression for the state reconstruction by using the results of all possible collective measurements stored in $\widetilde{Q}_{\rho}(\mathbf{m})$. The "tomographic representation" (60) is incomplete since the map (26) is not faithful. In other words, Eq.(60) should be considered as a form of arranging the information obtained from $\sim N^{d^{2}-1}$ collective measurements (corresponding to the total number of multiplets $\{\mathbf{m}\}$ ) in a $d^{N} \times d^{N}$ matrix. 
Equation (60) can be explicitly rewritten in terms of average values of symmetrized monomials (2) as follows

$$
\begin{aligned}
& \hat{\rho}_{\text {rec }}=d^{-N} \sum_{\mathbf{m}}\left(R_{\mathbf{m}}^{(d)}\right)^{-1}\left\langle\hat{D}_{\mathbf{m}}\right\rangle \hat{D}_{\mathbf{m}}^{\dagger}, \\
& \hat{D}_{\mathbf{m}}=\sum_{\alpha, \beta} \delta_{\mathbf{h}(\alpha, \beta), \mathbf{m}} Z_{\alpha} X_{\beta}, \quad\left\langle\hat{D}_{\mathbf{m}}\right\rangle=\operatorname{Tr}\left(\hat{\rho} \hat{D}_{\mathbf{m}}\right),
\end{aligned}
$$

which is a generalization of results obtained in [31]. The operators (62) can be always expressed in terms of polynomials of the collective operators (46)

$$
\hat{D}_{\mathbf{m}}=\sum_{\mathrm{p}} c_{\mathrm{p}}^{(\mathbf{m})} \prod_{k, l} O_{k, l}^{\mathrm{p} k, l}, \quad \sum_{k, l} \mathrm{p}_{k, l} \leq \sum_{k, l} m_{k l}
$$

(see Appendix D), and thus, accessed from Von Neumann measurements.

Since any state obtained from $\hat{\rho}$ by particle permutations, $\mathcal{P} \hat{\rho} \mathcal{P}^{\dagger}$, leads to the same $\hat{\rho}_{\text {rec }}$, the density matrix reconstructed according to Eq.(61) is related to the original one by the full symmetrization,

$$
\hat{\rho}_{\text {rec }}=\frac{1}{N !} \sum_{\mathcal{P}} \mathcal{P} \hat{\rho} \mathcal{P}^{\dagger} .
$$

Thus, the reconstruction (61) is exact for permutationally invariant (symmetric) states. It is straightforward to obtain a closed from expression for the reconstruction fidelity of a pure state $\hat{\rho}=|\psi\rangle\langle\psi|$,

$$
\mathcal{F}=\operatorname{Tr}\left(\hat{\rho} \hat{\rho}_{\text {rec }}\right)=\frac{1}{N !} \sum_{\mathcal{P}}|\langle\psi|\mathcal{P}| \psi\rangle|^{2},
$$

which reaches its minimum value

$$
\mathcal{F}_{\text {min }}=\frac{1}{N !}
$$

for states that become orthogonal under any particle permutation, e.g for $N$-qudit, $d \geq N$, elements of the computational basis $|\lambda\rangle=\left|l_{1}, \ldots, l_{N}\right\rangle$, with $l_{i} \neq l_{j}$.

For mixed states the minimum value of the fidelity can be substantiality smaller then given in Eq.(66). For instance, for 2 and 3 qubits one obtains $\mathcal{F}_{\min }=1 / 2$ and $\mathcal{F}_{\min }=1 / 8$ correspondingly; for 2 and 3 qutrits $\mathcal{F}_{\min }=1 / 9$ and $\mathcal{F}_{\min }=1 / 27$. 


\section{A. Symmetric space reconstruction}

In the particular case of fully symmetric states, the reconstruction expressions (601)-(61) become exact and can be rewritten in an explicit form by projecting the expansion (15) into the symmetric subspace.

Let us consider a symmetric density matrix, i.e. $\hat{\rho}_{s}=\hat{\Pi}_{s} \hat{\rho}_{s} \hat{\Pi}_{s}$, where $\hat{\Pi}_{s}$ is the projection operator on the symmetric subspace $\mathcal{H}_{\text {sym }}$ of $N$ qudits

$$
\hat{\Pi}_{s}=\sum_{\mathfrak{p}}|\mathfrak{p} ; N\rangle\langle\mathfrak{p} ; N|
$$

and permutationally invariant states

$$
\begin{aligned}
|\mathfrak{p} ; N\rangle & =\left|p_{1}, \ldots p_{d-1} ; N\right\rangle \\
p_{j} & =0, \ldots, N, \quad \sum_{j=1}^{d-1} p_{j} \leq N
\end{aligned}
$$

are elements of an orthonormal basis, $\left\langle\mathfrak{p}^{\prime}, N \mid \mathfrak{p}, N\right\rangle=\delta_{\mathfrak{p}^{\prime}, \mathfrak{p}}$, in $d_{\text {sym }}$-dimensional Hilbert space $\mathcal{H}_{\text {sym }}$,

$$
d_{\text {sym }}=\frac{(N+d-1) !}{(d-1) ! N !} .
$$

The states (68) are expanded in the computational basis according to

$$
|\mathfrak{p}, N\rangle=\mathcal{N}_{\mathfrak{p}} \sum_{\lambda} \prod_{i=1}^{d-1} \delta_{\eta_{i}^{(\lambda)}, p_{i}}|\lambda\rangle,
$$

where

$$
\eta_{i}^{(\lambda)}=\frac{(-1)^{i+1}}{(d-1-i) ! i !} \sum_{j=1}^{N} \prod_{\substack{k=0 \\ k \neq i}}^{d-1}\left(k-l_{j}\right),
$$

denotes the number of coefficients $l_{j}$ equal to $i=1, \ldots, d-1$ in the state $|\lambda\rangle=\left|l_{1}, \ldots, l_{N}\right\rangle$, and

$$
\mathcal{N}_{\mathfrak{p}}=\sqrt{\frac{p_{1} ! \ldots p_{d-1} !\left(N-p_{1}-\ldots-p_{d-1}\right) !}{N !}},
$$

is the normalization constant. It is easy to see that $\eta_{i}^{(\lambda)}$ are symmetric functions of $\lambda$, $\eta_{i}^{(\lambda)}=\eta_{i}(h(\lambda))$.

A density matrix $\hat{\rho}_{s}$ can be represented according to Eq. (15) as follows

$$
\hat{\rho}_{s}=\sum_{\alpha, \beta} \operatorname{tr}\left(\hat{\rho}_{s} \hat{\Delta}_{s}^{(-1)}(\alpha, \beta)\right) \hat{\Delta}_{s}^{(1)}(\alpha, \beta),
$$


where $\hat{\Delta}_{s}^{( \pm 1)}(\alpha, \beta)$ are the kernels (103) projected into the symmetric subspace,

$$
\hat{\Delta}_{s}^{( \pm 1)}(\alpha, \beta)=\hat{\Pi}_{s} \hat{\Delta}^{( \pm 1)}(\alpha, \beta) \hat{\Pi}_{s} \equiv \hat{\Delta}_{s}^{( \pm 1)}(\mathbf{h}(\alpha, \beta))
$$

acting in $\mathcal{H}_{\text {sym }}$.

The first-rank projectors

$$
\hat{\Delta}_{s}^{(-1)}(\mathbf{h}(\alpha, \beta))=\left|\phi_{\mathbf{h}(\alpha, \beta)}\right\rangle\left\langle\phi_{\mathbf{h}(\alpha, \beta)}\right|
$$

where

$$
\begin{aligned}
\left|\phi_{\mathbf{h}(\alpha, \beta)}\right\rangle & =\hat{\Pi}_{s}|\alpha, \beta\rangle=\sum_{\mathfrak{p}} \mathcal{N}_{\mathfrak{p}} \Upsilon_{\mathfrak{p}}(\mathbf{h}(\alpha, \beta))|\mathfrak{p}, N\rangle \\
\Upsilon_{\mathfrak{p}}(\mathbf{h}(\alpha, \beta)) & =\sum_{\lambda} \omega^{\alpha \lambda} c_{\lambda-\beta} \prod_{i=1}^{d-1} \delta_{\eta_{i}^{(\lambda)}, p_{i}},
\end{aligned}
$$

being $c_{\mu}$ the fiducial state expansion coefficients (40), define the measurement sets and satisfy the completeness condition

$$
\sum_{\mathbf{m}} \hat{E}_{\mathbf{m}}^{(d)}=\hat{\Pi}_{s}, \quad \hat{E}_{\mathbf{m}}^{(d)}=d^{-N} R_{\mathbf{m}}^{(d)} \hat{\Delta}_{s}^{(-1)}(\mathbf{h}(\alpha, \beta)=\mathbf{m})
$$

where $R_{\mathbf{m}}^{(d)}$ is defined in $(29)$ and $\hat{\Pi}_{s}$ acts as an identity operator in $\mathcal{H}_{\text {sym }}$. After a straightforward algebra one can show that the $\hat{\Delta}_{s}^{(1)}$ - kernel (75) in the basis (마) acquires the form

$$
\left\langle\mathfrak{t}, N\left|\hat{\Delta}_{s}^{(1)}(\mathbf{m})\right| \mathfrak{t}^{\prime}, N\right\rangle=\sum_{\mathfrak{t}, \mathfrak{t}^{\prime}} \mathcal{N}_{\mathfrak{t}} \mathcal{N}_{\mathfrak{t}^{\prime}}\left[\sum_{\mathbf{q}} g^{(d)}(\mathbf{q}, \mathbf{m}) f^{(d)}(\mathbf{q}) C_{\mathfrak{t}, \mathfrak{t}^{\prime}}(\mathbf{q})\right]
$$

where

$$
\begin{gathered}
g^{(d)}(\mathbf{q}, \mathbf{m})=\sum_{\alpha, \beta} \omega^{\alpha \delta-\beta \gamma} \delta_{\mathbf{h}(\alpha, \beta), \mathbf{m}} \delta_{\mathbf{h}(\gamma, \delta), \mathbf{q}}, \\
C_{\mathfrak{t}, \mathfrak{t}^{\prime}}(\mathbf{q})=\sum_{\gamma, \delta, \lambda} \omega^{\gamma \lambda} \prod_{i=1}^{d-1} \delta_{\eta_{i}^{(\lambda)}, t_{i}} \prod_{j=1}^{d-1} \delta_{\eta_{j}^{(\lambda-\delta)}, t_{j}^{\prime}} \delta_{\mathbf{h}(\gamma, \delta), \mathbf{q}}
\end{gathered}
$$

are the special discrete functions (special functions of discrete variables) and the coefficient

$$
f^{(d)}(\mathbf{q})=\sum_{\gamma, \delta}\left(\left\langle\xi\left|Z_{\gamma} X_{\delta}\right| \xi\right\rangle\right)^{-1} \delta_{\mathbf{h}(\gamma, \delta), \mathbf{q}}=\left.R_{\mathbf{q}}^{(d)}\left(\left\langle\xi\left|Z_{\gamma} X_{\delta}\right| \xi\right\rangle\right)^{-1}\right|_{\mathbf{h}(\gamma, \delta)=\mathbf{q}}
$$

can be always evaluated analytically, see (106). 
Finally, the explicit reconstruction expression in the symmetric subspace $\mathcal{H}_{\text {sym }}$ acquires the form

$$
\hat{\rho}_{s}=d^{N} \sum_{\mathbf{m}} \sigma_{\mathbf{m}}\left(R_{\mathbf{m}}^{(d)}\right)^{-1} \hat{\Delta}_{s}^{(1)}(\mathbf{m})
$$

where

$$
\sigma_{\mathbf{m}}=\operatorname{Tr}\left(\hat{E}_{\mathbf{m}}^{(d)} \hat{\rho}_{s}\right), \quad \sum_{\mathbf{m}} \sigma_{\mathbf{m}}=1
$$

are measured probabilities. The total number of projections required in this protocol is given by Eq.(31), while the density matrix of a fully symmetric state contains at most $d_{\text {sym }}^{2}-1$ independent parameters. Such a redundancy occurs because the probabilities (85) are not linearly independent and satisfy the following self-consistence conditions

$$
\sigma_{\mathbf{q}}=d^{N} \sum_{\mathbf{m}} \sigma_{\mathbf{m}}\left(R_{\mathbf{m}}^{(d)}\right)^{-1}\left\langle\phi_{\mathbf{q}}\left|\hat{\Delta}_{s}^{(1)}(\mathbf{m})\right| \phi_{\mathbf{q}}\right\rangle
$$

In order to estimate the accuracy of the reconstruction scheme (84) we numerically studied the minimum square error (MSE) of the quadratic Hilbert-Schmidt distance between a real state $\hat{\rho}_{s}$ and its estimate $\hat{\rho}_{\text {est }}$ according to the Crámer-Rao lower bound [32] for qubit and qutrit states, see Appendix E.

1. In N-qubit case, $d=2$, a single index (72) defines symmetric states,

$$
\eta_{1}^{(\lambda)}=\sum_{i=1}^{N} l_{i}=h(\lambda) .
$$

The elements of the basis in the symmetric subspaces are the well known Dicke states [33]

$$
\begin{aligned}
\left|p_{1} ; N\right\rangle & =\mathcal{N}_{p_{1}} \sum_{\lambda} \delta_{h(\lambda), p_{1}}|\lambda\rangle \\
\mathcal{N}_{p_{1}} & =\sqrt{\frac{p_{1} !\left(N-p_{1}\right) !}{N !}} .
\end{aligned}
$$

The symmetrized discrete coherent states (77) are expanded in the Dicke basis as follows

$$
\begin{aligned}
\left|\phi_{\mathbf{h}(\alpha, \beta)}\right\rangle & =\sum_{p_{1}} \mathcal{N}_{p_{1}} \Upsilon_{p_{1}}(\mathbf{h}(\alpha, \beta))\left|p_{1} ; N\right\rangle \\
\Upsilon_{p_{1}}(\mathbf{h}(\alpha, \beta)) & =\left(1+|\zeta|^{2}\right)^{-N / 2} \sum_{\lambda}(-1)^{\alpha \lambda} \zeta^{h(\lambda+\beta)} \delta_{h(\lambda), p_{1}}
\end{aligned}
$$


where $\zeta$ is defined in Eq. (42). The functions (81) and (8ㅏ) have the form

$$
\begin{aligned}
g^{(2)}(\mathbf{q}, \mathbf{m}) & =\sum_{\alpha, \beta}(-1)^{\alpha \delta+\beta \gamma} \delta_{\mathbf{h}(\alpha, \beta), \mathbf{m}} \delta_{\mathbf{h}(\gamma, \delta), \mathbf{q}} \\
f^{(2)}(\mathbf{q}) & =3^{\frac{q_{10}+q_{01}+q_{11}}{4}} i^{\frac{q_{11}-q_{10}-q_{01}}{2}} R_{\mathbf{q}}^{(2)}
\end{aligned}
$$

where $h(\gamma)=q_{10}, h(\delta)=q_{01}, h(\gamma+\delta)=q_{11}$ and

$$
C_{\mathfrak{t}, \mathfrak{t}^{\prime}}(\mathbf{q})=\sum_{\gamma, \delta, \lambda}(-1)^{\gamma \lambda} \delta_{h(\lambda), t_{1}} \delta_{h(\lambda-\delta), t_{1}^{\prime}} \delta_{h(\gamma), q_{10}} \delta_{h(\delta), q_{01}} \delta_{h(\gamma+\delta), q_{11}}
$$

can be expressed in terms of ${ }_{4} F_{3}$ - functions.

We have numerically found that the MSE $\sqrt{\left\langle\left\langle\mathcal{E}_{\min }^{2}\right\rangle\right\rangle}$, averaged over 200 pure and mixed states, is inversely proportional to the square root of the number of trials $M$,

$$
\sqrt{\left\langle\left\langle\mathcal{E}_{\min }^{2}\right\rangle\right\rangle} \approx \frac{\lambda}{\sqrt{M}}
$$

In Fig. 3 we plot the proportionality constant $\lambda$ for pure and mixed states for $N=1, \ldots, 6$ qubits and compare with the values corresponding to the SIC POVM tomographic protocol [27].

2. In N-qutrit case, $d=3$, there are two indexes (72) that define qutrit symmetric space,

$$
\begin{aligned}
& \eta_{1}^{(\lambda)}=\sum_{i=1}^{N} l_{i}\left(2-l_{i}\right)=\frac{2}{3}\left[h(2 \lambda)-\frac{1}{2} h(\lambda)\right]=p_{1}, \\
& \eta_{2}^{(\lambda)}=\sum_{i=1}^{N} \frac{l_{i}\left(l_{i}-1\right)}{2}=\frac{2}{3}\left[h(\lambda)-\frac{1}{2} h(2 \lambda)\right]=p_{2},
\end{aligned}
$$

thus, the basis in the symmetric subspace has the form

$$
\begin{aligned}
\left|p_{1}, p_{2} ; N\right\rangle & =\mathcal{N}_{p_{1} p_{2}} \sum_{\lambda} \delta_{h(\lambda), p_{1}+2 p_{2}} \delta_{h(2 \lambda), 2 p_{1}+p_{2}}|\lambda\rangle \\
\mathcal{N}_{p_{1} p_{2}} & =\sqrt{\frac{p_{1} ! p_{2} !\left(N-p_{1}-p_{2}\right) !}{N !}}
\end{aligned}
$$

The symmetrized discrete coherent states (77) are

$$
\begin{aligned}
\left|\phi_{\mathbf{h}(\alpha, \beta)}\right\rangle & =\sum_{p_{1}, p_{2}} \mathcal{N}_{p_{1} p_{2}} \Upsilon_{p_{1} p_{2}}(\mathbf{h}(\alpha, \beta))\left|p_{1}, p_{2} ; N\right\rangle, \\
\Upsilon_{p_{1} p_{2}}(\mathbf{h}(\alpha, \beta)) & =\sum_{\lambda} \omega^{\alpha \lambda} c_{\lambda-\beta} \delta_{h(\lambda), p_{1}+2 p_{2}} \delta_{h(2 \lambda), 2 p_{1}+p_{2}},
\end{aligned}
$$




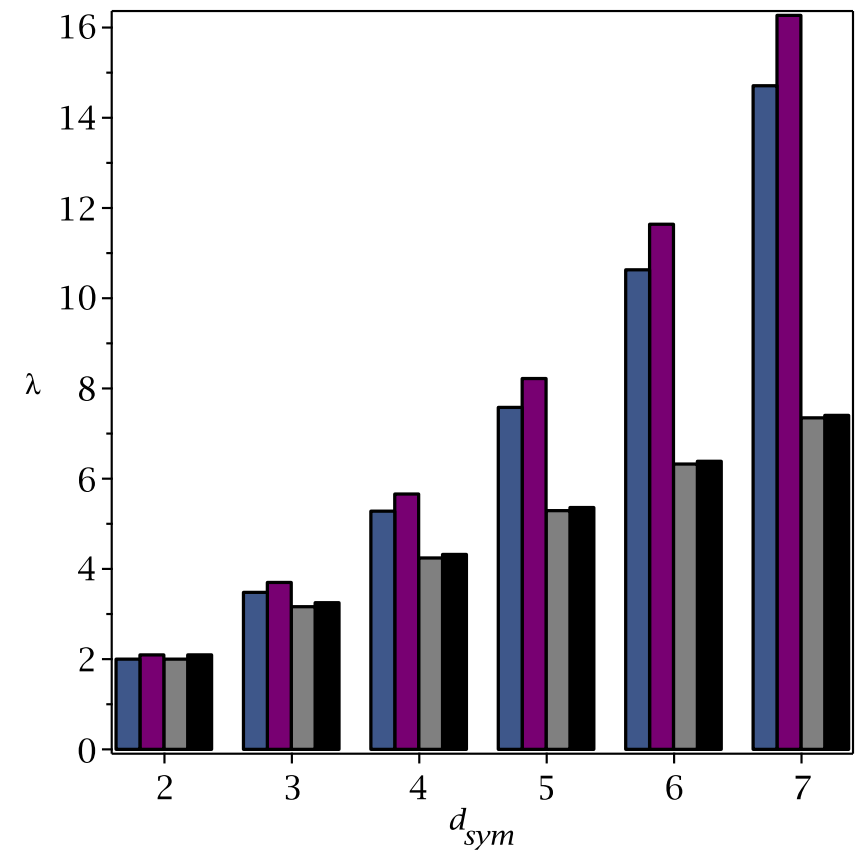

FIG. 3: First two columns: the proportionality constant $\lambda$ appearing in the minimum square error (93) for pure (left column) and mixed (right column) states $d_{\text {sym }}=N+1$ for $N=1, \ldots, 6$ qubits corresponding to the reconstruction skill (84); Second two columns: the corresponding values of $\lambda$ for SIC tomographic protocol.

where $\omega=\exp (2 \pi i / 3)$ and the $c_{\lambda}$ correspond to the fiducial state (45). The matrix element in Eq. (83) is

$$
\left.\left(\left\langle\xi\left|Z_{\gamma} X_{\delta}\right| \xi\right\rangle\right)^{-1}\right|_{\mathbf{h}(\gamma, \delta)=\mathbf{q}}=2^{\frac{1}{9} \sum_{k, l} q_{k l}-N} \mathrm{e}^{\frac{i \pi}{9}\left(q_{21}-q_{22}+q_{12}+5 q_{11}-9 q_{01}-3\left(q_{10}-q_{02}+q_{20}\right)\right)},
$$

where $q_{k l}=h(k \gamma+l \delta)$ and

$$
C_{t_{1} t_{2} t_{1}^{\prime} t_{2}^{\prime}}(\mathbf{q})=\sum_{\gamma, \delta, \lambda} \omega^{\gamma \lambda} \delta_{\mathbf{h}(\gamma, \delta), \mathbf{q}} \delta_{h(\lambda), t_{1}+2 t_{2}} \delta_{h(2 \lambda), 2 t_{1}+t_{2}} \delta_{h(\lambda-\delta), t_{1}^{\prime}+2 t_{2}^{\prime}} \delta_{h(2 \lambda-2 \delta), 2 t_{1}^{\prime}+t_{2}^{\prime}}
$$

We have numerically found that for qutrits the minimum error $\sqrt{\left\langle\left\langle\mathcal{E}_{\text {min }}^{2}\right\rangle\right\rangle}$ also behaves according to Eq. (93). In Fig. 4 we plot the constant $\lambda$ for pure and mixed qutrit states, $N=1,2,3$. 


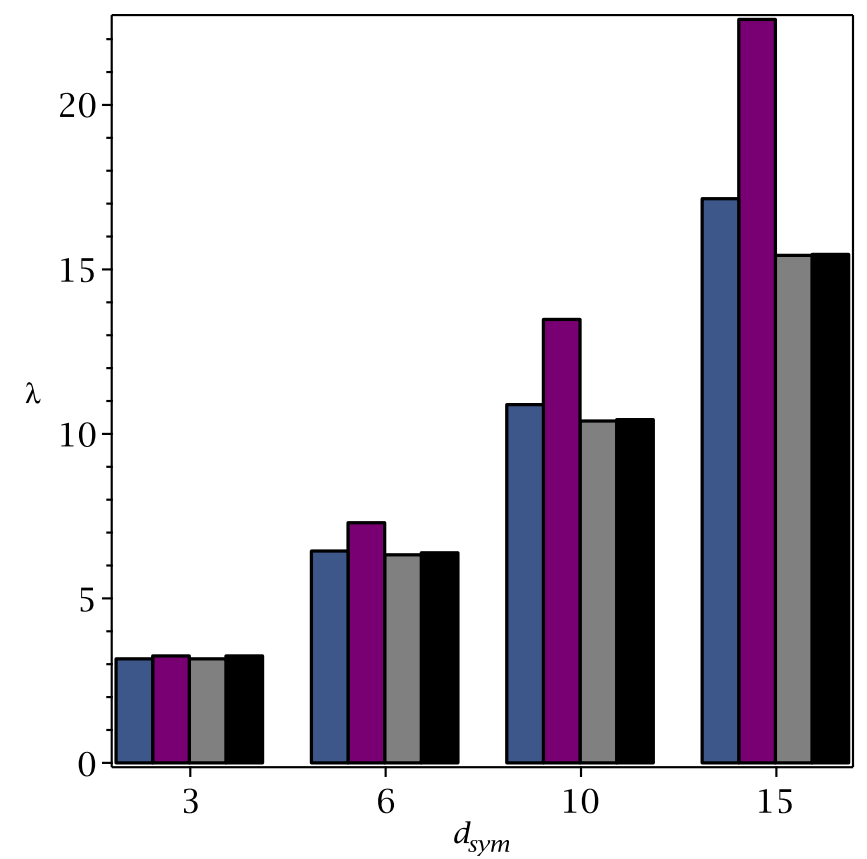

FIG. 4: First two columns: the proportionality constant $\lambda$ appearing in the minimum square error (93) for pure (left column) and mixed (right column) states $d_{\text {sym }}=(N+1)(N+2) / 2$ for $N=1, \ldots, 4$ qubits corresponding to the reconstruction skill (84); Second two columns: the corresponding values of $\lambda$ for SIC tomographic protocol.

\section{B. Conclusions}

We have developed a general framework for the analysis of $N$-qudit systems in the macroscopic limit, which includes:

a) A scheme to organize the information obtained from collective measurements in form of distributions functions in a discrete low-dimensional space spanned by the symmetric functions (21). The analysis of these projected $\tilde{Q}$-functions provides a useful insight into global properties of multipartite quantum states. It allows, for instance, to identify the relevant set of collective measurements for a given state, which are not always obvious from the state decomposition in the computational basis.

b) A set of collective operators appropriate for characterization of $N$-qudit states. The algebraic properties (49)-(50) of these operators (46) allow their efficient measurements with further applications to the collective tomography protocol (61);

c) Explicit expressions for the state reconstruction from collective measurements, both 
in the whole $d^{N}$-dimensional space (60), (61) and in the symmetric subspace (84). The symmetric tomographic protocol based on projective measurements performed in POVM (176) is characterized by a larger MSE than the optimal SIC reconstruction scheme for Nqubit states. Nevertheless, for larger $d$ - dimensions the relative difference between both reconstruction methods becomes smaller. The advantage of the proposed method consists in the possibility to generate the first rank collective POVM (76) for $N$ qudit systems in a systematic way for an arbitarary number of particles.

A deeper analysis of the projected $N$-qudit $\tilde{Q}$-function and their applications is in progress and will be published elsewhere.

This work is partially supported by the Grant 254127 of CONACyT (Mexico).

we outline the general method for computing $P$-symbols of collective operators.

Consider a generic (non-Hermitian) collective operator

$$
\sum_{i=1}^{N} Z_{i}^{m} X_{i}^{n}=\hat{s}_{m n}, m, n \in Z_{d} .
$$

Any other collective operator can be constructed from (102). Taking into account the explicit form of the kernels (7)

$$
\begin{array}{r}
\hat{\Delta}^{(\mp 1)}(\alpha, \beta)=\frac{1}{d^{\frac{1}{2}(3 \pm 1) N}} \sum_{\gamma, \delta} \omega^{\alpha \delta-\beta \gamma-\frac{1}{2}(1 \pm 1) \gamma \delta}\left(\left\langle\xi\left|Z_{\mp \gamma} X_{\mp \delta}\right| \xi\right\rangle\right)^{ \pm 1} Z_{\gamma} X_{\delta}, \\
\operatorname{Tr} \hat{\Delta}^{(\mp 1)}(\alpha, \beta)=d^{\frac{1}{2}(1 \mp 1) N}, \sum_{\alpha, \beta} \hat{\Delta}^{(\mp 1)}(\alpha, \beta)=d^{\frac{1}{2}(1 \pm 1) N} \hat{I},
\end{array}
$$

the $P$-function of (102) is represented as follows according to (7)

$$
P_{m n}=\frac{\omega^{m n}}{d^{N}}\left(\left\langle\xi\left|Z^{-m} X^{-n}\right| \xi\right\rangle\right)^{-1} \sum_{i=1}^{N} \omega^{m b_{i}-n a_{i}} .
$$

Observe, that for the fiducial state of the form (12)-(14) the matrix element $\left\langle\xi\left|Z_{\gamma} X_{\delta}\right| \xi\right\rangle$ is symmetric function of its argument,

$$
\left\langle\xi\left|Z_{\gamma} X_{\delta}\right| \xi\right\rangle=\left.\left\langle\xi\left|Z_{\gamma} X_{\delta}\right| \xi\right\rangle\right|_{\mathbf{h}(\gamma, \delta)=\mathbf{p}}
$$

The sum $\sum_{i} \omega^{m b_{i}-n a_{i}}$ that appear in the above equation in can be rewritten as

$$
\begin{aligned}
& \sum_{i=1}^{N} \omega^{m b_{i}-n a_{i}}=\sum_{i=1}^{N}\left[\frac{1}{(d-1) ! b_{i}}+\frac{\omega^{m}}{(d-2) !\left(1-b_{i}\right)}+\frac{\omega^{2 m}}{-2(d-3) !\left(2-b_{i}\right)}+\ldots\right] \\
& {\left[\frac{1}{(d-1) ! a_{i}}+\frac{\omega^{-n}}{(d-2) !\left(1-a_{i}\right)}+\frac{\omega^{-2 n}}{-2(d-3) !\left(2-a_{i}\right)}+\ldots\right] \Pi_{k=0}^{d-1}\left(k-b_{i}\right)\left(k-a_{i}\right),}
\end{aligned}
$$


which is always reduced to the form

$$
\sum_{i=1}^{N} a_{i}^{p} b_{i}^{q}=\sum_{k, l=0}^{d-1} B_{k, l}^{(p, q)} h(k \alpha+l \beta)
$$

where $B_{k, l}^{(p, q)}$ are some coefficients. Thus, the $P$-function (105) is a function of the weights (21).

\section{Examples}

a) Qubits, $d=2$

The sum (107) takes the form, $\omega=-1$

$$
\sum_{i=1}^{N} \omega^{m b_{i}-n a_{i}}=N-2 \delta_{m, 0} \delta_{n, 1} h(\alpha)-4 \delta_{m, 1} \delta_{n, 0} h(\beta)-4 \delta_{m, 1} \delta_{n, 1} h(\alpha+\beta)
$$

so that, for the fiducial state (42)

$$
P_{m n}(\alpha, \beta)=\frac{\sqrt{3}}{2^{N}}\left[N-2\left(h(\alpha) \delta_{m, 0} \delta_{n, 1}+h(\beta) \delta_{m, 1} \delta_{n, 0}+h(\alpha+\beta) \delta_{m, 1} \delta_{n, 1}\right)\right]
$$

b) Qutrits, $d=3$.

The sum (107) is significantly more complicated, $\omega=\exp (2 \pi i / 3)$,

$$
\begin{gathered}
\sum_{i=1}^{N} \omega^{m b_{i}-n a_{i}}=N-\left[\delta_{m, 0} \delta_{n, 1} e^{-i \pi / 3}+\delta_{m, 0} \delta_{n, 2} e^{i \pi / 3}\right] h(\alpha)-\left[\delta_{m, 0} \delta_{n, 1} e^{i \pi / 3}+\delta_{m, 0} \delta_{n, 2} e^{-i \pi / 3}\right] h(2 \alpha) \\
-\left[\delta_{m, 1} \delta_{n, 0} e^{i \pi / 3}+\delta_{m, 2} \delta_{n, 0} e^{-i \pi / 3}\right] h(\beta)-\left[\delta_{m, 1} \delta_{n, 0} e^{-i \pi / 3}+\delta_{m, 2} \delta_{n, 0} e^{i \pi / 3}\right] h(2 \beta) \\
-\left[\delta_{m, 1} \delta_{n, 2} e^{i \pi / 3}+\delta_{m, 2} \delta_{n, 1} e^{-i \pi / 3}\right] h(\alpha+\beta)-\left[\delta_{m, 1} \delta_{n, 2} e^{-i \pi / 3}+\delta_{m, 2} \delta_{n, 1} e^{i \pi / 3}\right] h(2 \alpha+2 \beta) \\
-\left[-\delta_{m, 1} \delta_{n, 1} e^{i \pi / 3}+\delta_{m, 2} \delta_{n, 2} e^{-i \pi / 3}\right] h(2 \alpha+\beta)-\left[\delta_{m, 1} \delta_{n, 1} e^{-i \pi / 3}+\delta_{m, 2} \delta_{n, 2} e^{i \pi / 3}\right] h(\alpha+2 \beta) .
\end{gathered}
$$

The matrix elements of the operators (46) have the form

$$
\begin{array}{r}
{ }_{i}\left\langle p\left|\hat{O}_{0, l}^{(i)}\right| q\right\rangle_{i}=\delta_{p, q}\left[1-\frac{2}{(d-1)} \sum_{r=0}^{d-1}\{l r\}\left|c_{p-r}^{(i)}\right|^{2}\right] \\
{ }_{i}\left\langle p\left|\hat{O}_{\lambda, \lambda m}^{(i)}\right| q\right\rangle_{i}=\delta_{p, q}-\frac{2 \tau_{q-p, \lambda}}{d(d-1)} \sum_{r=0}^{d-1} \omega^{-m r(q-p)} c_{p-r}^{(i)} c_{q-r}^{(i) *}
\end{array}
$$

where $c_{p}^{(i)}$ are the expansion coefficients (39), and $\tau_{q, \lambda}=\sum_{r=0}^{d-1} r \omega^{r q \lambda^{-1}}, \lambda, m=0, \ldots, d-1$. The trace of two operators (46) has the form 


$$
\begin{aligned}
\operatorname{tr}\left(\hat{O}_{k, l} \hat{O}_{k^{\prime}, l^{\prime}}\right) & =N^{2} d^{N}-\frac{2 N}{(d-1) d^{N}}\left(\sum_{\alpha, \beta} h(k \alpha+l \beta)+\sum_{\alpha, \beta} h\left(k^{\prime} \alpha+l^{\prime} \beta\right)\right) \\
+\frac{4}{(d-1)^{2} d^{2 N}} \frac{1}{d^{N}+1} & \left(\sum_{\alpha, \beta, \alpha^{\prime}, \beta^{\prime}} h(k \alpha+l \beta) h\left(k^{\prime} \alpha^{\prime}+l^{\prime} \beta^{\prime}\right)-\sum_{\alpha, \beta} h(k \alpha+l \beta) h\left(k^{\prime} \alpha+l^{\prime} \beta\right)\right) \\
& +\frac{4}{(d-1)^{2} d^{2 N}} \sum_{\alpha, \beta} h(k \alpha+l \beta) h\left(k^{\prime} \alpha+l^{\prime} \beta\right),
\end{aligned}
$$

where we have used the SIC POVM condition (14).

Taking into account that

$$
\begin{aligned}
\sum_{\mu} h(\mu) & =\frac{N(d-1) d^{N}}{2}, \\
\sum_{\alpha, \beta} h(k \alpha+l \beta) & =\frac{N(d-1) d^{2 N}}{2}
\end{aligned}
$$

we obtain

$$
\operatorname{tr}\left(\hat{O}_{k, l} \hat{O}_{k^{\prime}, l^{\prime}}\right)=-\frac{N^{2} d^{N}}{d^{N}+1}+\frac{4}{d^{N}(d-1)^{2}\left(d^{N}+1\right)} \sum_{\alpha, \beta} h(k \alpha+l \beta) h\left(k^{\prime} \alpha+l^{\prime} \beta\right) .
$$

In case when $k \neq \lambda k^{\prime}$ and $l \neq \lambda l^{\prime}, \lambda=1, \ldots, d-1$ we can use new variables $k \alpha+l \beta=\mu$, $\lambda\left(k^{\prime} \alpha+l^{\prime} \beta\right)=\nu$ and sum over the new variables $\mu, \nu$ so that the last sum in (115) is just a square of (113) and thus

$$
\operatorname{tr}\left(\hat{O}_{k, l} \hat{O}_{k^{\prime}, l^{\prime}}\right)=0
$$

if $k=\lambda k^{\prime}$ and $l=\lambda l^{\prime}$, then

$$
\operatorname{tr}\left(\hat{O}_{k, l} \hat{O}_{k^{\prime}, l^{\prime}}\right)=-\frac{N^{2} d^{N}}{d^{N}+1}+\frac{4}{(d-1)^{2}\left(d^{N}+1\right)} \sum_{\mu} h(\mu) h(\lambda \mu) .
$$

Taking into account the representation (15) and the form of the P-function (47)

$$
\begin{gathered}
\hat{O}_{k, l}=\sum_{\alpha, \beta} \frac{1}{d^{N}}\left[N-\frac{2}{d-1} h(k \alpha+l \beta)\right] \Delta^{(-1)}(\alpha, \beta) \\
=N \hat{I}-\frac{2}{d^{2 N}(d-1)} \sum_{\gamma, \delta} \omega^{-\gamma \delta}\left[\sum_{\alpha, \beta} h(k \alpha+l \beta) \omega^{\alpha \delta-\beta \gamma}\right]\left\langle\xi\left|Z_{-\gamma} X_{-\delta}\right| \xi\right\rangle Z_{\gamma} X_{\delta}
\end{gathered}
$$


we immediately observe that

$$
\left[\hat{O}_{k, 0}, \hat{O}_{\lambda k, 0}\right]=\left[\hat{O}_{0, l}, \hat{O}_{0, \lambda l}\right]=0
$$

If $k \neq 0, l \neq 0$ for $d>2$ then after a change of variables

$$
\begin{gathered}
\alpha=(\mu+\nu)(2 k)^{-1}, \\
\beta=(\mu-\nu)(2 l)^{-1},
\end{gathered}
$$

where $(2 k)^{-1}$ is understood as the inverse element in $Z_{d}$, we represent $\hat{O}_{k, l}$ as

$$
\hat{O}_{k, l}=N \hat{I}-\frac{2}{d^{N}(d-1)} \sum_{\gamma} \omega^{k l^{-1} \gamma^{2}}\left[\sum_{\mu} h(\mu) \omega^{-\mu \gamma l^{-1}}\right]\left\langle\xi\left|Z_{-\gamma} X_{k l^{-1} \gamma}\right| \xi\right\rangle Z_{\gamma} X_{-k^{-1} \gamma l}
$$

so that

$$
\left[\hat{O}_{k, l}, \hat{O}_{\lambda k, \lambda l}\right]=0 \text {. }
$$

It is worth noting that the average values of the collective operators (46) in the states (77) are proportional to the corresponding $P$-symbols (47)

$$
\left\langle\phi_{\mathbf{h}(\alpha, \beta)=\mathbf{m}}\left|\hat{O}_{k, l}\right| \phi_{\mathbf{h}(\alpha, \beta)=\mathbf{m}}\right\rangle=\frac{d^{N}}{d+1} P_{\hat{O}_{k, l}}
$$

Here we present explicit expressions for $R_{\mathbf{q}}^{(d)}$-functions for qubits and qutrits:

$$
\begin{gathered}
R_{\mathbf{q}}^{(2)}=\frac{N !}{\left(\frac{q_{10}+q_{01}-q_{11}}{2}\right) !\left(\frac{2 N-q_{10}-q_{01}-q_{11}}{2}\right) !\left(\frac{q_{10}-q_{01}+q_{11}}{2}\right) !\left(\frac{q_{01}+q_{11}-q_{10}}{2}\right) !}, \\
0 \leq q_{k l}=h(k \gamma+l \delta) \leq N
\end{gathered}
$$


and

$$
\begin{aligned}
&\left(R_{\mathbf{q}}^{(3)}\right)^{-1}=\frac{1}{N !}\left(N-\frac{q_{10}+q_{20}+q_{01}+q_{02}+q_{11}+q_{22}+q_{21}+q_{12}}{9}\right) ! \times \\
&\left(\frac{2 q_{10}-q_{20}+2 q_{01}-q_{02}-q_{11}+2 q_{22}-q_{21}-q_{12}}{9}\right) ! \times \\
&\left(\frac{2 q_{10}-q_{20}-q_{01}+2 q_{02}-q_{11}-q_{22}+2 q_{21}-q_{12}}{9}\right) ! \times \\
&\left(\frac{2 q_{10}-q_{20}-q_{01}-q_{02}+2 q_{11}-q_{22}-q_{21}+2 q_{12}}{9}\right) ! \times \\
&\left(\frac{-q_{10}+2 q_{20}+2 q_{01}-q_{02}-q_{11}-q_{22}-q_{21}+2 q_{12}}{9}\right) ! \times \\
&\left(\frac{-q_{10}+2 q_{20}-q_{01}+2 q_{02}+2 q_{11}-q_{22}-q_{21}-q_{12}}{9}\right) ! \times \\
&\left(\frac{-q_{10}+2 q_{20}-q_{01}-q_{02}-q_{11}+2 q_{22}+2 q_{21}-q_{12}}{9}\right) ! \times \\
&\left(\frac{-q_{10}-q_{20}+2 q_{01}-q_{02}+2 q_{11}-q_{22}+2 q_{21}-q_{12}}{9}\right) ! \times \\
&\left(\frac{-q_{10}-q_{20}-q_{01}+2 q_{02}-q_{11}+2 q_{22}-q_{21}+2 q_{12}}{9}\right) ! \\
& 0 \leq q_{k l}=h(k \gamma+l \delta) \leq N
\end{aligned}
$$

In the limit $N \gg 1$ the function $R_{\mathbf{q}}^{(d)}$ tends to a Gaussian form localized in the vicinity of the point $\mathbf{q}_{0}=\frac{d-1}{2}(N, \ldots, N)$, for instance,

$$
\begin{gathered}
R_{q_{10} q_{01} q_{11}}^{(2)} \sim \exp \left(-2\left(\mathbf{q}-\mathbf{q}_{0}\right)^{2} / N\right), \\
R_{\mathbf{q}}^{(3)} \sim \exp \left(-\sum_{i=0}^{1} \sum_{j=0}^{i+1} \frac{\left[\left(q_{i j}-N\right)^{2}+\left(q_{2 i 2 j}-N\right)^{2}-\left(q_{i j}-N\right)\left(q_{2 i 2 j}-N\right)\right]}{N}\right) .
\end{gathered}
$$

Any product of collective operators (46) can be directly expanded in the monomial basis (4):

$$
\prod_{k, l} O_{k, l}^{\mathrm{p} k, l}=\sum_{\gamma, \delta} b_{\mathbf{h}(\gamma, \delta), \mathrm{p}} Z_{\gamma} X_{\delta}
$$

The required representation of the symmetrized monomials in terms of measurables operators (46)

$$
\sum_{\mu, \nu} Z_{\mu} X_{\nu} \delta_{\mathbf{h}(\mu, \nu), \mathbf{m}}=\sum_{\mathrm{p}} C_{\mathrm{p}}^{(\mathbf{m})} \prod_{k, l} O_{k, l}^{\mathrm{p}_{k, l}}, \quad \sum_{k, l} \mathrm{p}_{k, l} \leq \sum_{k, l} m_{k l}
$$


is thus obtained by substituting (128) into (129) and comparing the coefficients of the same basis elements. This leads to the following set of equations for the coefficients $C_{\mathrm{p}}^{(\mathbf{m})}$

$$
\delta_{\mathbf{h}(\mu, \nu), \mathbf{m}}=\sum_{\mathrm{p}} C_{\mathrm{p}}^{(\mathbf{m})} b_{\mathbf{h}(\mu, \nu), \mathrm{p}} .
$$

In case of qubits, (128) adquires the following form for diagonal operators

$$
\sum_{\mu} Z_{\mu} \delta_{h(\mu), k}=\sum_{p=0}^{k} C_{p}^{(k)} S_{z}^{p}
$$

Taking into account that the collective spin operator in the computational basis has the form

$$
S_{z}=\sum_{\nu}(N-h(\nu))|\nu\rangle\langle\nu|,
$$

we arrive at the following expression for the coefficient $b$

$$
b_{h(\mu), p}=\frac{1}{2^{N}} \sum_{\nu}(N-h(\nu))^{p} \chi(\mu \nu)=\frac{1}{2^{N}} \sum_{m=0}^{N}\left(\begin{array}{l}
N \\
m
\end{array}\right)(N-m)^{p} g^{(2)}(m),
$$

where

$$
g^{(2)}(m)=\sum_{\nu}(-1)^{\mu \nu} \delta_{h(\nu), m},
$$

is a discrete special function, which can be expressed in terms of $P_{n}^{(\alpha, \beta)}(z)$ Jacobi polynomials. The system (130) takes the form

$$
\delta_{h(\mu), m}=\sum_{p \leq m} C_{p}^{(k)} b_{h(\mu), p}
$$

leading in particular to

$$
\begin{aligned}
& \sum_{\mu} Z_{\mu} \delta_{h(\mu), 1}=\hat{S}_{z}, \\
& \sum_{\mu} Z_{\mu} \delta_{h(\mu), 2}=\hat{S}_{z}^{2}-N \hat{I} .
\end{aligned}
$$

Similar calculations can be carried out for qutrits:

$$
\sum_{\mu} Z_{\mu} \delta_{h(\mu), m_{1}} \delta_{h(2 \mu), m_{2}}=\sum_{p_{1}+p_{2} \leq m_{1}+m_{2}} C_{p_{1}, p_{2}}^{\left(m_{1}, m_{2}\right)} \hat{O}_{0,1}^{p_{1}} \hat{O}_{0,2}^{p_{2}}
$$


where

$$
\hat{O}_{0, l}^{p}=\sum_{\nu}\left(N-\sum_{\varepsilon} h(l \varepsilon)\left|c_{\nu-\varepsilon}\right|^{2}\right)^{p}|\nu\rangle\langle\nu|,
$$

being $c_{\nu}$ expansion coefficients (40) of the fiducial state in the computational basis.

Thus the coefficients $b$ have the form

$$
\begin{aligned}
b_{\mathbf{h}(\mu), p_{1}, p_{2}} & =\frac{1}{3^{N} 2^{p_{1}+p_{2}}} \sum_{m_{1}, m_{2}}\left(m_{2}-m_{1}\right)^{p_{1}}\left(N-m_{1}\right)^{p_{2}} g^{(3)}\left(m_{1}, m_{2}\right) \\
g^{(3)}\left(m_{1}, m_{2}\right) & =\sum_{\nu} \delta_{h(\nu), m_{1}} \delta_{h(2 \nu), m_{2}} \omega^{-\mu \nu}, \omega=\exp (2 \pi i / 3),
\end{aligned}
$$

where we used the explicit expansion (45). The set of equations (130) to be inverted is now

$$
\delta_{\mathbf{h}(\mu), \mathbf{m}}=\sum_{p_{1}+p_{2} \leq m_{1}+m_{2}} C_{p_{1}, p_{2}}^{\left(m_{1}, m_{2}\right)} b_{\mathbf{h}(\mu), p_{1}, p_{2}} .
$$

In particular, we find,

$$
\begin{aligned}
& \sum_{\mu} Z_{\mu} \delta_{h(\mu), 1}=2 \omega \hat{O}_{0,1}+2 \hat{O}_{0,2} \\
& \sum_{\mu} Z_{\mu} \delta_{h(\mu), 2}=4\left(\omega \hat{O}_{0,1}+\hat{O}_{0,2}\right)^{2}+2\left(\omega^{2} \hat{O}_{0,1}+\hat{O}_{0,2}\right) .
\end{aligned}
$$

The performance of a reconstruction scheme can be measured by the statistical average of the Hilbert-Schmidt distance between the real $\rho_{s}$ and estimated $\tilde{\rho}_{s}$ states,

$$
\left\langle\mathcal{E}^{2}\right\rangle=\left\langle\operatorname{Tr}\left[\left(\rho_{s}-\tilde{\rho}_{s}\right)^{2}\right]\right\rangle
$$

when only a finite number of copies $(M)$ are involved in the measurement process [34].

The multinomial measurement statistics associated to the protocol (84)

$$
\mathcal{P} \propto \prod_{\mathbf{p}} \tilde{\sigma}_{\mathbf{p}}^{n_{\mathbf{p}}}, \quad \sum_{\mathbf{p}} n_{\mathbf{p}}=M
$$

allows to relate the estimated probabilities $\tilde{\sigma}_{\mathbf{p}}$ with the corresponding frequencies $n_{\mathbf{p}} / M$, according to

$$
\left\langle n_{\mathbf{p}}\right\rangle=M \tilde{\sigma}_{\mathbf{p}},\left\langle n_{\mathbf{p}}^{2}\right\rangle=M \tilde{\sigma}_{\mathbf{p}}\left(1+(M-1) \tilde{\sigma}_{\mathbf{p}}\right),\left\langle n_{\mathbf{p}} n_{\mathbf{q}}\right\rangle=M(M-1) \tilde{\sigma}_{\mathbf{p}} \tilde{\sigma}_{\mathbf{q}}
$$


Using the explicit reconstruction form (84) we obtain the square error in terms of the deviation between probabilities $\sigma_{\mathbf{p}}$ and their estimates $\tilde{\sigma}_{\mathbf{p}}$,

$$
\left\langle\mathcal{E}^{2}\right\rangle=\sum_{\mathbf{p}, \mathbf{q}} \mathcal{A}_{\mathbf{p}, \mathbf{q}} \Delta \sigma_{\mathbf{p}} \Delta \sigma_{\mathbf{q}}
$$

where $\Delta \sigma_{\mathbf{p}}=\sigma_{\mathbf{p}}-\tilde{\sigma}_{\mathbf{p}}$ and

$$
\mathcal{A}_{\mathbf{p}, \mathbf{q}}=d^{2 N}\left(R_{\mathbf{p}}^{(d)} R_{\mathbf{q}}^{(d)}\right)^{-1} \operatorname{Tr}\left(\hat{\Delta}_{s}^{(1)}(\mathbf{p}) \hat{\Delta}_{s}^{(1)}(\mathbf{q})\right) .
$$

Taking into account the redundancy (86) and normalization (85) conditions we obtain the square error in terms of the deviation between the independent probabilities $\sigma_{\mathbf{p}}^{\prime}$ and their estimates $\tilde{\sigma}_{\mathbf{p}}^{\prime}$

$$
\left\langle\mathcal{E}^{2}\right\rangle=\sum_{\mathbf{p}^{\prime}, \mathbf{q}^{\prime}} \mathcal{A}_{\mathbf{p}^{\prime} \mathbf{q}^{\prime}}^{\prime} \Delta \sigma_{\mathbf{p}^{\prime}} \Delta \sigma_{\mathbf{q}^{\prime}},
$$

where the sum is taken only on the indexes $\mathbf{p}^{\prime}, \mathbf{q}^{\prime}$ labeling the independent probabilities; the coefficients $\mathcal{A}_{\mathbf{p}^{\prime} \mathbf{q}^{\prime}}^{\prime}$ are not explicitly given here due to of their cumbersome form.

The minimum square error (MSE) is given by the Cramér-Rao bound [32],

$$
\left\langle\mathcal{E}^{2}\right\rangle \geq \operatorname{Tr}\left(\mathcal{A}^{\prime} \mathcal{F}^{-1}\right)
$$

were $\mathcal{A}^{\prime}$ is $\left(d_{\text {sym }}^{2}-1\right) \times\left(d_{\text {sym }}^{2}-1\right)$ matrix with the elements $\mathcal{A}_{\mathbf{p}^{\prime} \mathbf{q}^{\prime}}^{\prime}$, while $\mathcal{F}$ is the Fisher matrix for the independent probabilities,

$$
\mathcal{F}_{\mathbf{p}^{\prime} \mathbf{q}^{\prime}}=\left\langle\frac{\partial \ln \mathcal{P}}{\partial \sigma_{\mathbf{p}^{\prime}}^{\prime}} \frac{\partial \ln \mathcal{P}}{\partial \sigma_{\mathbf{q}^{\prime}}^{\prime}}\right\rangle .
$$

To estimate the average minimum square error we numerically compute the statistical mean value over the symmetric states,

$$
\sqrt{\left\langle\left\langle\mathcal{E}_{\text {min }}^{2}\right\rangle\right\rangle}=\sqrt{\left\langle\operatorname{Tr}\left(\mathcal{A}^{\prime} \mathcal{F}^{-1}\right)\right\rangle}
$$

\section{References}

[1] Gross D, Liu Y K, Flammia S T, Becker S and Eisert 2010 Phys. Rev. Lett. 105 15040; Liu W T, Zhang T, Liu J Y, Chen P X and Yuan J M 2012 Phys. Rev. Lett. 108 170403; S Straupe 2016 JETP Lett. 104, 7 
[2] Häffner $\mathrm{H}$ et al 2005 Nature 438 643; Cramer M, Plenio M B, Flammia S T, Somma R, Gross D, Bartlett S D, Landon-Cardinal O, Poulin D and Liu Y K 2010 Nat. Commun. 1 149; Baumgratz T, Gross D, Cramer M and Plenio M B 2013 Phys. Rev. Lett. 111 020401; Qi B, Hou Z, Li L, Dong D, Xiang G and Guo G 2013 Sci. Rep. 33496

[3] R B A Adamson and A M Steinberg 2010 Phys. Rev. Lett. 105 030406; Yin J O S and van Enk S J 2011 Phys. Rev. A 83062110

[4] D F V James, P G Kwiat, W J Munro, and A G White 2001 Phys. Rev. A 64 052312;Paris M G A and J Řeháček J, 2004 Quantum State Estimation (Berlin Heidelberg:Springer) 649;J Nunn, B J Smith, G Puentes, I A Walmsley, and J S Lundeen 2010 Phys. Rev. A 81042109

[5] W K Wootters 1987 Ann. Phys. 176 1; K S Gibbons, M J Hoffman, and W K Wootters 2004 Phys. Rev. A 70062101

[6] J P Paz, A J Roncaglia, and M Saraceno 2005 Phys. Rev. A 72 012309; C Cormick, E F Galvao, D Gottesman, J P Paz and A O Pittenger 2006 Phys. Rev. A 73 012301; I Bengtsson and K Zyczkowski Geometry of Quantum States: An Introduction to Quantum Entanglement Cambridge University Press 2008

[7] C Muñoz, A B Klimov, and L L Sanchez-Soto 2012 J. Phys. A 45 244014; A B Klimov and C Muñoz 2013 Phys. Scr. 87038110

[8] A B Klimov, C Munoz, and L L Sánchez-Soto 2009 Phys. Rev. A 80 043836; Muñoz C, Klimov A B and Sanchez-Soto L L 2012 J. Phys. A 45244014

[9] O Hosten, N Engelsen, R Krishnakumar, et al 2016 Nature 529 505; J Léonard, A Morales, P Zupancic, et al 2017 Nature 54387

[10] G Tóth, W Wieczorek, D Gross, R Krischek, C Schwemmer, and H Weinfurter 2010 Phys. Rev. Lett. 105 250403; T Moroder, P Hyllus, G Tóth, C Schwemmer, A Niggebaum, S Gaile, O Gühne, and H Weinfurter 2012 New J. Phys. 14 105001; Schwemmer C, Tóth G, Niggebaum A, Moroder T, Gross D, Gühne O, and Weinfurter H 2014 Phys. Rev. Lett. 113 040503; Zhibo Hou et al 2016 New J. Phys. 18083036

[11] A B Klimov and C Muñoz 2014 Phys. Rev. A 89052130

[12] M Gaeta, C Muñoz, and A B Klimov 2016 Phys. Rev. A 93062107

[13] P Rungta, et al 1999 Qudit entanglement, In Directions in Quantum Optics: A Collection of Papers Dedicated to Dan Walls (Springer-Verlag, Berlin, 2000), p. 149; N J Cerf, M Bourennane, A Karlsson, and N Gisin 2002 Phys. Rev. Lett. 88 127902; T Durt, D Kaszlikowski, J L 
Chen, and L C Kwek 2004 Phys. Rev. A 69 032313; D P O’Leary, G K Brennen, and Stephen S Bullock 2006 Phys. Rev. A 74 032334; G Vitagliano, I Apellaniz, I L Egusquiza, G Toth 2014 Phys. Rev. A 89 032307; T Kraft, C Ritz, N Brunner, M Huber, and O Gühne 2018 Phys. Rev. Lett. 120060502

[14] R T Thew, K Nemoto, A G White, and W J Munro 2002 Phys. Rev. A 66012303

[15] D Giovannini, J Romero, J Leach, A Dudley, A Forbes, and M J Padgett 2013 Phys. Rev. Lett. 110143601

[16] R Bianchetti, S Filipp, M Baur, J M Fink, C Lang, L Steffen, M Boissonneault, A Blais, and A Wallraff 2010 Phys. Rev. Lett. 105 223601; H Sosa-Martinez, N K Lysne, C H Baldwin, A Kalev, I H Deutsch, and P S Jessen 2017 Phys. Rev. Lett. 119150401

[17] N K Langford, R B Dalton, M D Harvey, J L O'Brien, G J Pryde, A Gilchrist, S D Bartlett, and A G White 2004 Phys. Rev. Lett. 93, 053601

[18] W M PZ E D Medendorp, F A Torres-Ruiz, L K Shalm, G N M Tabia, C A Fuchs, and A M Steinberg 2011 Phys. Rev. A 83051801

[19] G Lima, L Neves, R Guzmán, E S Gómez, W A T Nogueira, A Delgado, A Vargas and C Saavedra 2011 Opt. Exp. 193542

[20] W M Pimenta, B Marques, T O Maciel, R O Vianna, A Delgado, C Saavedra, and S Pádua 2013 Phys. Rev. A 88012112

[21] J B Altepeter, E R Jefferey and P G Kwait 2005 Adv. At. Mol. Opt. Phys. 52 105; G Lima, L Neves, R Guzmán, E S Gómez, W A T Nogueira, A Delgado, A Vargas, C Saavedram 2011 Opt. Express 193542

[22] N Bent, H Qassim, A A Tahir, D Sych, G Leuchs, L L Sánchez-Soto, E Karimi, and R W Boyd 2015 Phys. Rev. X 5041006

[23] H Häner, W Hänsel, C F Roos, J Benhelm, D Chek-al kar, M Chwalla, T Körber, U D Rapol, M Riebe, P O Schmidt, C Becher, O Gühne, W Dür, and R Blatt 2005 Nature 438 7068; M Agnew, J Leach, M Mclaren, F Stef Roux, and R W Boyd 2011 Phys. Rev. A 84062101

[24] D Gottesman 1996 Phys. Rev. A 54 1862; E Hostens, J Dehaene, and B De Moor 2005 Phys. Rev. A 71 042315; A Vourdas 2004 Rep. Prog. Phys. 67 267; A B Klimov, C Muñoz and J L Romero 2006 J. Phys. A 3914471

[25] J Schwinger 1960 Proc. Natl. Acad. Sci. USA 46 570; ibid. 196046 883; ibid. 1960461401

[26] D Galetti, and M A Marchiolli 1996 Ann. Phys. 249 454; Ruzzi M, Marchiolli M A and Galetti 
D 2005 J. Phys. A 38 6239; Marchiolli M A, Ruzzi M and Galetti D 2005 Phys. Rev. A 72 042308.

[27] J M Renes, R Blume-Kohout, A J Scott, and C M Caves 2004 J. Math. Phys. (N.Y.) 45, 2171

[28] G L Mullen, D Panario, Handbook of Finite Fields (Discrete Mathematics and Its Applications) (CRC Press, Taylor and Francis group, 2013).

[29] M Gaeta, C Muñoz and A B Klimov 2018 J. Phys. A 51 115303; C Muñoz, M Gaeta, R Gomez, A B Klimov 2019 Phys. Rev. Lett. A 383141

[30] J Patera and H Zassenhaus 1988 J. Math. Phys. 29665

[31] A Muñoz, A B Klimov, M Grassl, . L Sanchez-Soto 2018 Quant. Infor. Proc. 17286

[32] C W Helstrom 1979 Quantum Detection and Estimation Theory ed R Bellman (Academic Press) Volume 123

[33] Dicke R 1954 Phys. Rev. A. 93, 99

[34] Z Bian, J Li, H Qin et al. 2015 Phys. Rev. Lett. 114 203602; Z Hou, J Tang, J Shang et al. 2018 Nat. Commun. 91414 